\title{
MORAR COM MAIS INDEPENDÊNCIA E AUTONOMIA: UMA REFLEXÃO SOB A PERSPECTIVA DO DESIGN
}

\author{
Luiza Beck Arigoni \\ Pontifícia Universidade Católica do Rio de Janeiro \\ luizaarigoni@hotmail.com \\ Marilia Ceccon \\ Universidade Federal do Rio de Janeiro \\ mariliaceccon@yahoo.com.br \\ Vera Damazio \\ Pontifícia Universidade Católica do Rio de Janeiro \\ vdamazio@puc-rio.br
}

Resumo: Dados estatísticos mostram que o Brasil está passando por uma inversão em sua pirâmide etária e junto com o envelhecimento o país experimenta, também, o aumento da expectativa de vida de sua população. Essa nova realidade exige atenção especial do Design no sentido de atender as demandas novas e crescentes daqueles que estão envelhecendo e vivendo cada vez mais. Nesse contexto, esse artigo explora meios mais adequados e acolhedores de moradia para idosos e está dividido em quatro partes: a primeira introduz dados sobre o envelhecimento populacional; a segunda aborda os conceitos de independência e autonomia; a terceira apresenta alguns arranjos domiciliares para maiores de 60 anos e a quarta, apresenta soluções projetuais que contemplam formas de morar que promovem a autonomia e independência do idoso.

Palavras-chave: design emocional, envelhecimento, moradia para idosos

\begin{abstract}
Statistics show that Brazil is undergoing a reversal in its age pyramid and along with the population aging, the country also experience an increase in life expectancy. This new reality requires special attention of Design in the sense of attending the also new and crescent demands of those who are getting old and living more. In this context, this article explores more appropriate and welcoming means for housing to the elderly and is divided into four parts: the first explores data on population aging; the second deals with the concepts of independence and autonomy; the third present house arrangements of the over 60 years old; and the fourth presents design solutions that consider ways of living that promote the autonomy and independence of the elderly.
\end{abstract}

Keywords: emotional design, aging, housing for elderly 


\section{INTRODUÇÃO}

Avanços socioeconômicos e de saúde pública diminuíram taxas de natalidade e de mortalidade precoce e nos brindaram com o acréscimo de uma média de 20 anos, em comparação com os 50 anos anteriores (WORLD HEALTH ORGANIZATION, 2015). Essa verdadeira "Revolução da Longevidade" vem transformando a maioria das pirâmides etárias ao redor do mundo (INTERNATIONAL LONGEVITY CENTER BRAZIL, 2015).

Estamos vivendo mais, mas não necessariamente melhor (WHO, 2015). Envelhecimento é sinônimo de mudanças em diferentes níveis. $O$ passar dos anos pressupõe alterações biológicas, psicológicas, funcionais e de papéis sociais e econômicos (MOTLIS, 1985). Não havendo planejamento em relação a essas mudanças, o triunfo da longevidade pode se revelar uma adversidade.

No Brasil, o envelhecimento populacional tem acontecido de maneira acelerada. Segundo o Instituto Brasileiro de Geografia Estatística (IBGE, 2015), a proporção de pessoas acima de 60 anos, que em 2004 era de 9,7\%, aumentou para 13,7\% em 10 anos. Levamos cerca de 20 anos para vivenciar uma realidade que a França levou 150 anos. Há quem ainda se surpreenda com a constatação de que o Brasil não é mais um país com população predominantemente jovem. As consequências da diferença nas relações de planejamento e velocidade de envelhecimento entre os dois países podem ser mensuradas: no que diz respeito ao bem-estar econômico e social da população idosa ao redor do mundo, a França se apresenta como a 16a melhor nação para envelhecer, enquanto o Brasil ocupa a 56a posição (HELPAGE INTERNATIONAL, 2015).

Esses dados revelam a urgência de ações voltadas para as novas e crescentes demandas do público idoso e sua qualidade de vida. Neste sentido, o presente trabalho trata de questões sobre envelhecimento e demandas relacionadas à moradia, a partir de levantamento bibliográfico multidisciplinar e de exemplos projetuais.

\section{INDEPENDÊNCIA E AUTONOMIA: DETERMINANTES PARA MORAR SÓ}

O passar dos anos atua sobre o corpo humano modificando morfologias e funções. O envelhecimento fisiológico pode ser entendido como um resultado das "alterações típicas e inevitáveis" (NERI, 2007, p.30) relacionadas à senescência, somadas aos comportamentos da pessoa durante todo o curso de vida, como alimentação, sedentarismo e consumo de tabaco ou álcool, que podem determinar um envelhecimento patológico, também conhecido como senilidade (BICALHO \& CINTRA, 2013; CURIATI et al., 2011; RIBEIRO \& COSENZA, 2013; NUNES, 2003).

A combinação de fatores internos e externos determina, em maior ou menor grau, declínios nas habilidades mentais e físicas de um indivíduo. Essas habilidades compõem a sua "capacidade intrínseca" (WHO, 2015) e servem como recursos para a tomada de decisões e a execução das ações planejadas. Pode-se dizer que a "capacidade intrínseca" é um composto de quatro sistemas principais: (1) a cognição, "capacidade mental de compreender e resolver problemas do cotidiano"; (2) o humor, "motivação necessária para as atividades e/ou participação social" (3) a mobilidade, "capacidade de deslocamento e de manipulação do meio" e (3) a comunicação, "capacidade de estabelecer um relacionamento produtivo com o meio" (MORAES \& CINTRA, 2013, p.298). 
Níveis de cognição e de humor determinam a "autonomia" do idoso, definida como a "capacidade individual de decisão e comando sobre as ações, estabelecendo e seguindo as próprias regras". Os níveis de mobilidade e de comunicação, por sua vez, determinam sua "independência", definida como a "capacidade de realizar algo com os próprios meios" (MORAES \& CINTRA, 2013, p.297).

Alterações nessas capacidades interferem em atividades de autocuidado e cuidado da casa, denominadas "atividades de vida diária". Elas são classificadas como "básicas", "instrumentais" e "avançadas".

As "atividades básicas de vida diária" são aquelas relacionadas ao autocuidado, como banhar-se, alimentar-se e fazer uso de banheiro. As "atividades instrumentais de vida diária" são mais complexas que as básicas e referem-se ao cuidado com a casa, como a realização de trabalhos domésticos e à capacidade do idoso de viver sozinho. As "atividades avançadas de vida diária", por sua vez, referem-se a práticas recreativas, sociais, religiosas, laborais, entre outras relacionadas à sociabilidade (ASSIS, ASSIS \& CARDOSO, 2013; MORAES \& CINTRA, 2013).

A OMS prevê que em países em desenvolvimento, como é o caso do Brasil, o número de idosos que necessitam de ajuda para atividades de vida diária quadruplique até 2050 (WHO, 2015).

\section{RESILIÊNCIA E CAPACIDADE FUNCIONAL: A IMPORTÂNCIA DE SER E FAZER O QUE SE VALORIZA}

Um diferencial das publicações da Organização Mundial da Saúde em relação a outras bibliografias da área da saúde é o fato de não apenas considerarem recursos intrínsecos, mas também os extrínsecos, nos quais um indivíduo pode se apoiar durante a vida. Seguindo essa perspectiva, foi lançado no segundo semestre de 2015 o Relatório Mundial de Envelhecimento e Saúde (World Report on Ageing and Health).

A OMS defende que a complexidade dos estados de saúde na velhice demanda uma abordagem mais holística do conceito de saúde, que tenha como foco o funcionamento e o bem-estar dessas pessoas. Neste sentido, instiga uma mudança de paradigma ao conceituar o "envelhecimento saudável" como um processo dinâmico de desenvolvimento e manutenção da "capacidade funcional", que permite o bem-estar na velhice (WHO, 2015).

No relatório são definidos e discernidos dois conceitos-chaves: "capacidade intrínseca" e "capacidade funcional". O primeiro, já mencionado anteriormente, se refere à totalidade de características mentais e físicas as quais um indivíduo fará uso em diferentes fases da vida e que são influenciadas pelas alterações relacionadas ao envelhecimento (WHO, 2015).

Apesar de essas alterações serem, em geral, caracterizadas como declínios, elas não impedirão que as pessoas tenham qualidade de vida, se fizerem uso de recursos intrínsecos e extrínsecos que lhes proporcionem resistência, recuperação e adaptação. Esses recursos ajudam a manter ou maximizar a "capacidade funcional" e constituem o que denomina-se "resiliência".

O conceito de "capacidade funcional" resume a visão holística defendida pela OMS. Ela abrange a "capacidade intrínseca", o ambiente no qual o idoso está inserido e as interações entre eles. Abrange, em resumo, todos os recursos intrínsecos e extrínsecos que permitem o indivíduo ser e fazer o que preza e julga importante (WHO, 2015). 


\section{ALGUNS DADOS SOBRE MORADORES IDOSOS E SOBRE A CASA: UMA NOVA PERSPECTIVA PARA O DESIGN}

Vem crescendo o número de idosos morando sozinhos no Brasil como indicam os dados da Pesquisa Nacional por Amostra em Domicílios (PNAD). Enquanto em 1992, 1,17 milhão de idosos moravam sozinhos, em 2012 que esse número triplicou, chegando a cerca de 3,7 milhões (IBGE, 2012). Já o número de idosos morando em instituições de longa permanência é de aproximadamente 83 mil, número que pode ser considerado muito baixo, comparado ao de idosos que optam por continuar a morar sozinhos em suas casas (IPEA, 2011).

Há muitas explicações para esse movimento no Brasil e no mundo. Segundo a PNAD, as mudanças na constituição familiar são grandes responsáveis por essa alteração estatística. Os organizadores da pesquisa relacionaram o aumento da proporção de domicílios compostos por mulheres sozinhas com a transformação do papel social da mulher e o envelhecimento da população. Ressaltaram ainda, que as alterações na estrutura etária levaram ao aumento da proporção de mulheres viúvas, que podem estar optando por morar sozinhas em vez de morar com os filhos (PNAD, 2014).

Para o gerontólogo Alexandre Kalache, uma das razões para o aumento de idosos morando sozinhos pode ser a maior dispersão e fragmentação da família, com filhos muitas vezes morando em cidades ou até países diferentes. Ele argumenta, ainda, que os idosos querem manter a autonomia, principalmente as mulheres que já criaram os filhos e ficaram viúvas.

Podemos constatar também uma mudança cultural na composição familiar e consequentemente nos arranjos domiciliares. De acordo com a antropóloga Mirian Goldenberg (2013), na cultura brasileira ainda prevalece a imagem da família como fonte de segurança e felicidade. Nesse sentido, para muitos, a decisão de ter filhos está relacionada a ter amparo e companhia na velhice. O idoso que mora com outra pessoa, seja um familiar ou não, passa a imagem de estar mais bem assistido (RAMOS, MENEZES \& MEIRA, 2010). Goldemberg resume que: "A imagem de velho sozinho é associada ao abandono, desamparo, fracasso, insegurança. Os filhos são vistos como uma possível proteção contra a velhice solitária e infeliz, uma espécie de seguro contra velhice desamparada." No entanto, como revela o relatório da PNAD: "Durante milênios, prosperidade e felicidade eram associados a uma descendência ou a um legado para a prosperidade (PRITCHEET E VIARENGO, 2012). Hoje, prosperidade e felicidade caminham em direção opostas a uma família numerosa" (PNAD, 2014).

Ainda que continue prevalecendo uma forte valorização da família no processo de envelhecimento em nossa cultura, o número de idosos que tomam a decisão de morar sozinhos tem aumentado consideravelmente nas últimas décadas. Goldemberg (2015a) argumenta que a ideia de abandono e solidão em torno do fato de morar sozinho vem se desconstruindo em nosso país. A autora (2015b) acrescenta que hoje é possível se observar, também, uma mudança de significado da casa para o brasileiro de lugar para dormir e comer para espaço de lazer, prazer e criatividade. Com base em seus estudos de campo, Goldenberg conta que ouviu de muitos de seus entrevistados que suas casas eram um verdadeiro paraíso e espaço de prazer e de amor e conclui: 
Hoje, pode-se dizer que morar sozinho, em diferentes fases da vida, tornou-se uma opção legítima, especialmente para uma geração que está inventando uma nova forma de envelhecer, mais bela, prazerosa e significativa (GOLDENBERG, 2015b).

Em estudo realizado com o objetivo de levantar demandas do público idoso, constatou-se que quando os idosos se aposentam, eles passam mais tempo em casa não por falta de opção e sim por prazer. A maioria dos entrevistados demonstrou gostar muito de estar em casa. Muitos revelaram preferir passar a maior parte do tempo em casa porque nela se sentem mais seguros e confortáveis (CECCON, NOGUEIRA e DAMÁZIO, 2015).

Neste sentido a casa assume uma função que vai além da ideia de abrigo. Csikszentmihalyi e Rochberg-Halton (1981, p.9) ensinam que "a casa é o lugar de memórias passadas, experiências presentes, e sonhos futuros de cada pessoa. E eles são inseparáveis dos objetos que compõem seu ambiente". Os autores resumem que "a casa é muito mais do que um abrigo; é um mundo no qual uma pessoa pode criar um ambiente material que incorpora o que ele ou ela considera significativo. Sendo assim, a casa torna-se o sinal mais poderoso da identidade de seu morador" (CSIKSZENTMIHALYI E ROCHBERG-HALTON, 1981, p.123).

No livro A Memória Coletiva, Halbwachs (1990) dá ainda mais sentido à relação das pessoas com o espaço, quando nos apresenta ideia formulada por Auguste Comte, segundo a qual o equilíbrio mental decorreria, em boa parte, do fato de os objetos do nosso cotidiano mudarem pouco e nos oferecerem uma imagem de permanência e estabilidade. Halbwachs explica que os objetos são como uma sociedade silenciosa e imóvel, estranha a nossa agitação e mudanças de humor e, como tal, responsáveis pela transmissão das sensações de ordem e quietude. Reconhece, assim, a existência de uma forte relação entre as pessoas e as coisas que lhes são familiares, tal como demonstra seu comentário a respeito de como nos sentimos num meio estranho. "[...] antes de a ele nos adaptarmos, atravessamos um período de incerteza, como se houvéssemos deixado para trás toda a nossa personalidade (p.131)." O autor conclui que:

Nosso entorno material leva, ao mesmo tempo, nossa marca e a dos outros. Nossa casa, nossos móveis e a maneira segundo a qual estão dispostos, o arranjo dos cômodos aonde vivemos, lembram-nos nossa família e os amigos que víamos geralmente nesse quadro (HALBWACHS, 1990, P.131).

Diante do exposto, concluímos que a escolha por morar sozinho depende de fatores diversos e inclui desde questões pessoais e familiares a aspectos econômicos e culturais. Nenhum, contudo, parece ser mais desafiador do que o equilíbrio entre o desejo pessoal - ou querer morar sozinho - e as reduções na capacidade intrínseca relacionadas ao envelhecimento - ou conseguir morar sozinho. Estudos de orientação etnográfica tem revelado que muitos idosos preferem morar sozinhos a ir para instituições ou para a casa de filhos. Alguns revelaram preferir correr riscos a perder a privacidade (OMS, 2007; GOLDEMBERG, 2103; CECCON, 2015)

A necessidade de suporte físico, financeiro e afetivo faz com que muitos idosos acabem tendo como opção morar com familiares. Declínios nas capacidades cognitivas e de humor podem determinar perda de autonomia. Declínios nas capacidades de mobilidade e comunicação, por sua vez, perda de independência, resultando na 
necessidade de maior cuidado (MORAES \& CINTRA, 2007; CAMARGOS, RODRIGUES \& MACHADO, 2011). O Design, contudo, pode contribuir com o projeto de outras opções e novos arranjos domiciliares, como se pretende demonstrar a seguir.

\section{AÇÕES PROJETUAIS PARA MORAR COM MAIS INDEPENDÊNCIA E AUTONOMIA}

O Design está instrumentado para tratar de problemas complexos e transformar realidades existentes em outras mais desejáveis (FRASCARA, 2000). Como tal, a atividade apresenta enorme potencial para atender as mais diversas demandas dos maiores de 60 anos, incluindo a construção de espaços para moradia mais acolhedores, mais seguros, que potencializem os ganhos dessa fase da vida e minimizem suas limitações.

As iniciativas apresentadas a seguir são parte de acervo em construção a partir de método de busca na internet com cruzamento de palavras chaves relacionadas à moradia e envelhecimento saudável e tem como objetivo contribuir com a geração de soluções de moradia que preservam a autonomia e independência dos idosos. Elas contemplam meios de morar com mais significado, liberdade, privacidade e convívio social.

Vila ConViver: planejada pela GTMoradia/ADunicamp, é um conceito de cohousing, projeto para moradia de docentes da UniCamp com mais de 50 anos aposentados ou em vias de se aposentar. O projeto caracteriza-se pelo trabalho em grupo, onde seus futuros moradores participam da criação da vila. O modelo contempla tanto a privacidade como o convívio social em ambientes coletivos como pátios, jardins, cozinha e lavandeira. ${ }^{1}$

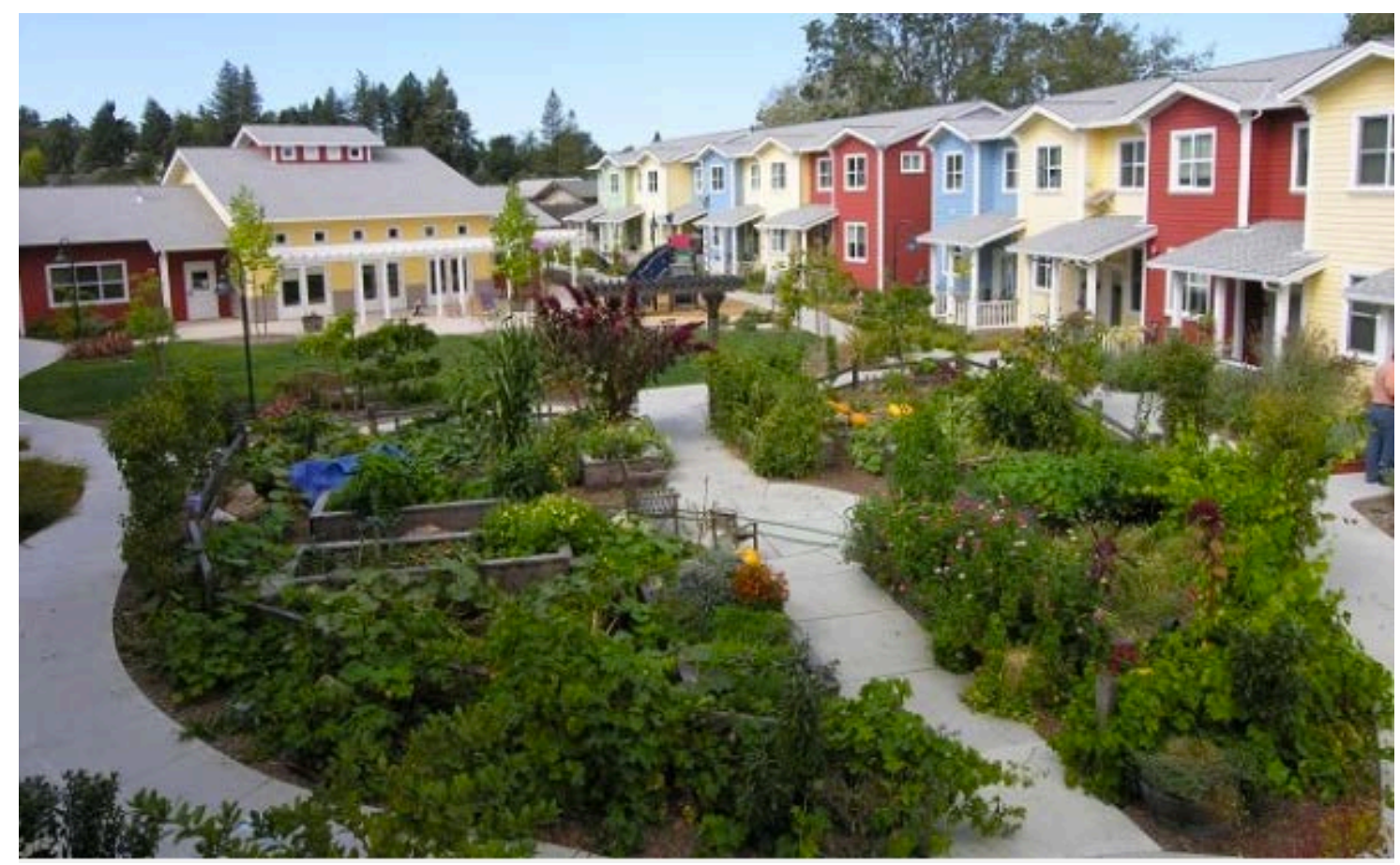

Figura 1 - Projeção 3D da Vila ConViver. ADUnicamp

\footnotetext{
${ }^{1}$ Diponível em: http://longevidadeadunicamp.org.br/?p=1959
} 
Vila Hogeweyk: localizada em Weesp na Holanda, foi projetada com o objetivo de promover a independência dos pacientes idosos com problemas de demência, principalmente Alzheimer, e faze-los se sentir em casa e não em uma instituição. 0 espaço reproduz as ruas e estabelecimentos comuns em um bairro - como casas, supermercado, restaurantes e parques - e conta com uma equipe de enfermeiros, cuidadores e médicos em todos os lugares. Os idosos moram em casas individuais ou com outros pacientes, fazem suas próprias compras no supermercado, saem para passear, visitam e jantam com os amigos. A equipe profissional não usa uniformes, assumindo o papel de vizinhos, prestadores de serviços, atendentes dos estabelecimentos, ajudante do lar etc. Além de preparada para atender as demandas de natureza mecânica e física dos pacientes, a equipe está orientada a estimula-los a falar de suas memórias e histórias. ${ }^{2}$

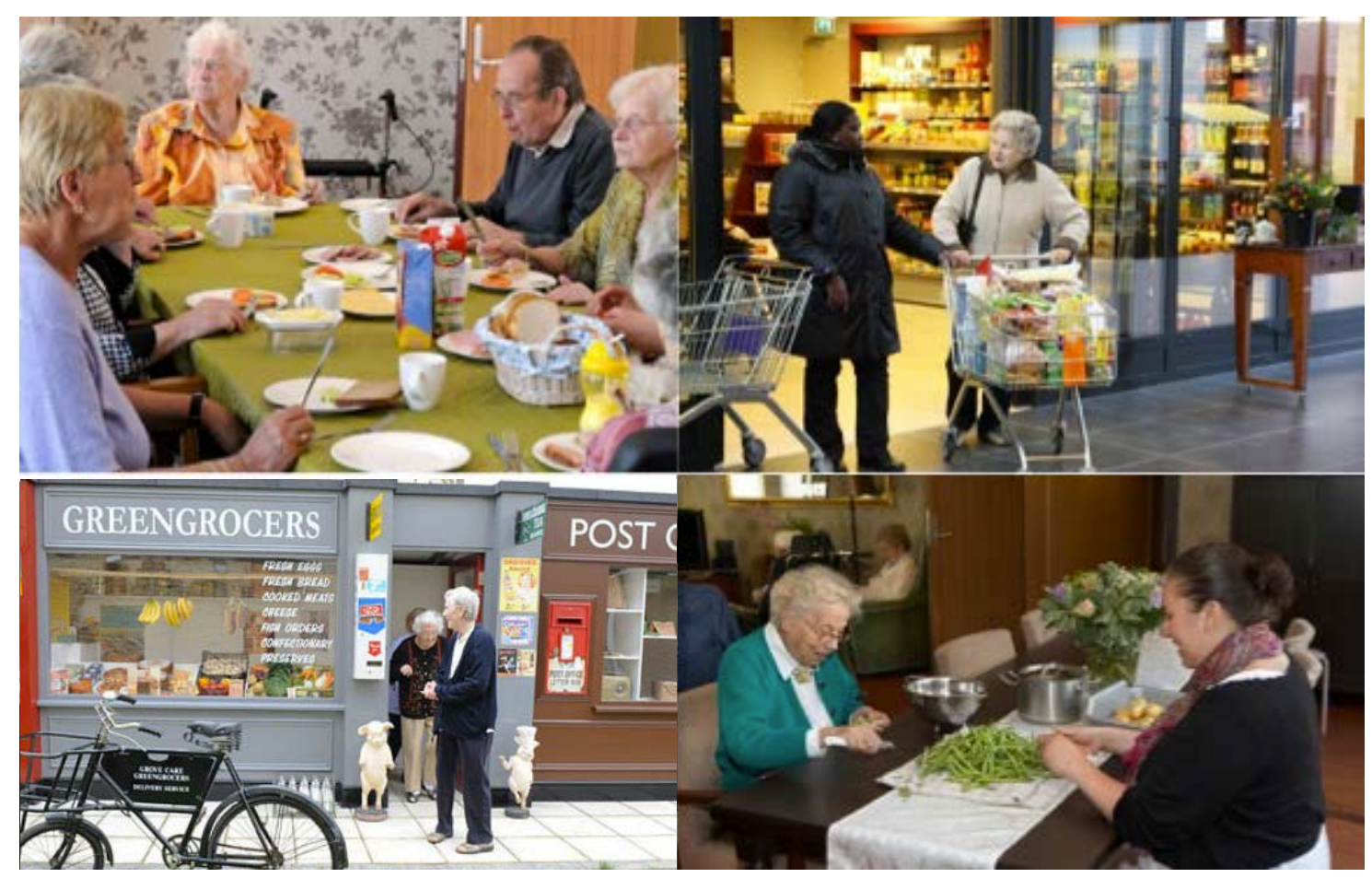

Figura 2 - Áreas coletivas da Vila Hogeweyk. twistedsifter.com

Home Farm: projeto conceitual criado pelo escritório Spark em Singapura com o objetivo de dar suporte aos idosos contemplando questões estruturais como adaptação das moradias, mas também de promover a geração de emprego e atividades de convívio social. No condomínio vertical para idosos, os moradores podem plantar sua própria horta e/ou trabalhar em outras funções durante algumas horas do dia. ${ }^{3}$

\footnotetext{
2 Disponível em: http://twistedsifter.com/2015/02/amazing-village-in-netherlands-just-for-people-withdementia/

${ }^{3}$ Disponível em: http://www.sparkarchitects.com/work/homefarm\#4
} 


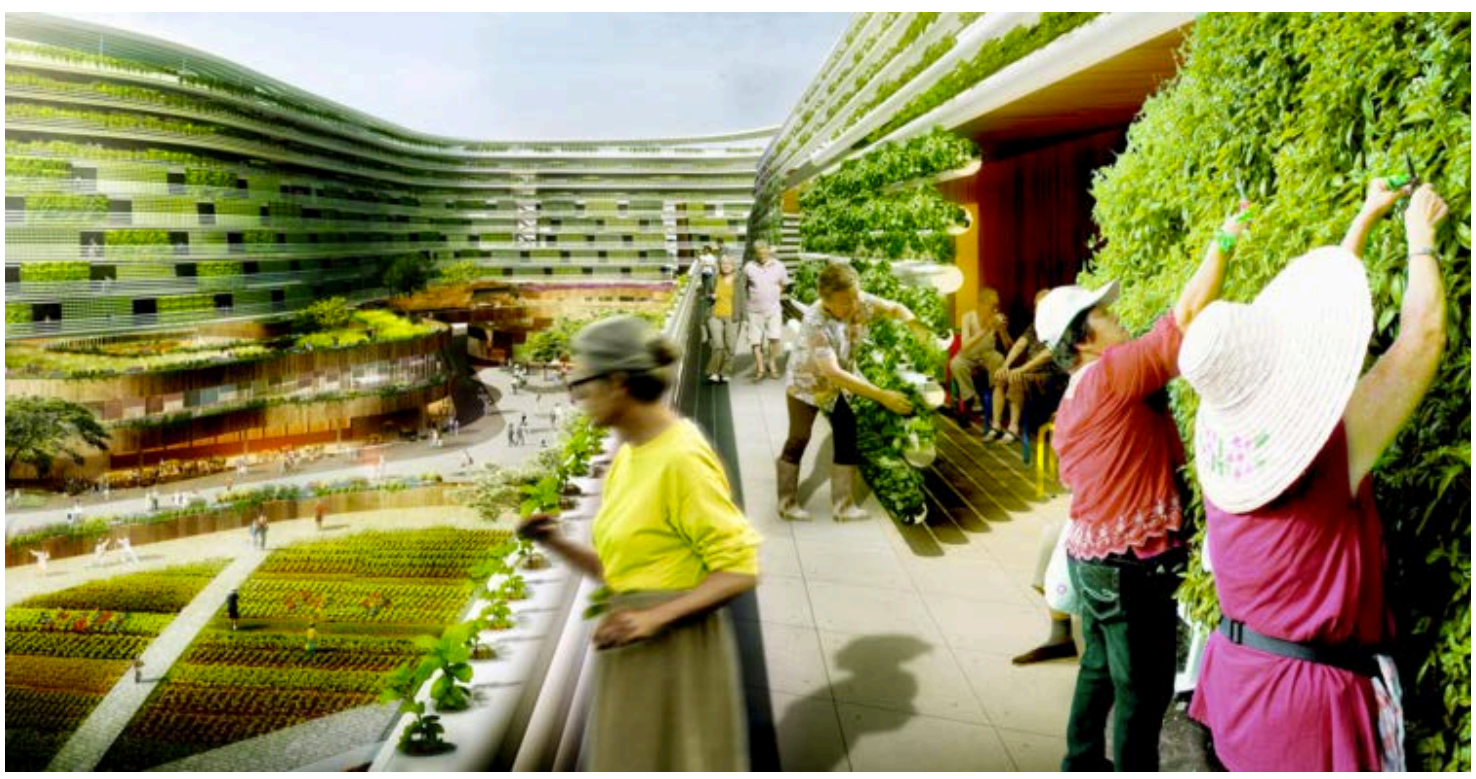

Figura 3 - simulação 3D da “Home Farm". Spark

\section{CONCLUSÃO}

Esse artigo buscou demonstrar a importância e urgência do Design dedicar esforços na investigação acerca de possibilidades do morar após os 60 anos, levando em consideração a promoção da autonomia e independência. Os resultados de esforços nesse sentido, decerto, contribuirão significativamente com a criação de meios mais adequados para essa parcela crescente da população não somente morar, mas viver de forma mais acolhedora e preservando sua individualidade e bem-estar. $E$ por último, mas não menos importante, faz-se necessário ressaltar que a velhice não é uma abstração, mas uma realidade vivida por cada vez mais pessoas e que todos deverão viver, ou deveriam almejar. Além de um problema complexo, a velhice é uma realidade que os leitores experimentam ou deverão experimentar pessoalmente e que muitas pessoas queridas já vivenciam. E o Design decerto haverá de trazer muitas contribuições para que se viva não apenas cada vez mais, mas cada vez melhor.

\section{REFERÊNCIAS}

ALVES, J; CAVANAGHI, S. Tendências demográficas, dos domicílios e das familias no Brasil. In: Aparte Inclusão Social em Debate, 2012. Disponível em: http://www.ie.ufrj.br/aparte/pdfs/tendencias_demograficas_e_de_familia_24ago12.p df Acesso em: 28 nov. 2014.

ASSIS, M. G.; ASSIS, L. O.; CARDOSO, A. P. Reabilitação das Tarefas Diárias. In: MALLOYDINIZ, L. F. I.; FUENTES, D.; COSENZA, R. M. (Org.) Neuropsicologia do Envelhecimento: Uma Abordagem Multidimensional. Porto Alegre: Artmed, 2013.

BICALHO, M. A. C.; CINTRA, M. T. G. Modificações Fisiológicas Sistêmicas no Envelhecimento. In: MALLOY-DINIZ, L. F. I.; FUENTES, D.; COSENZA, R. M. (Org.) Neuropsicologia do Envelhecimento: Uma Abordagem Multidimensional. Porto Alegre: Artmed, 2013. 
CAMARGOS, M.C.S., RODRIGUES, R.N. e MACHADO, C.J. Idoso, família e domicílio: uma revisão narrativa sobre a decisão de morar sozinho. In: R. bras. Est. Pop., Rio de Janeiro, v. 28, n. 1, p. 217-230, jan./jun. 2011. Disponível em: http://www.scielo.br/pdf/rbepop/v28n1/a12v28n1Acesso em: 15 jul. 2015

CECCON, M. ; NOGUEIRA, S. J ; DAMAZIO, V. M. M. . ENVELHECIMENTO ATIVO: novas perspectivas e oportunidades para o campo do design emocional. In: 11o P\&D Design 2014. Congresso Brasileiro de Pesquisa e Desenvolvimento em, 2014, Gramado, RS. 11o P\&D Design 2014. Congresso Brasileiro de Pesquisa e Desenvolvimento em Design.

CSIKSZENTMIHALYI, Mihaly; ROCHBERG-HALTON, Eugene. The Meaning of Things: Domestic Symbols and the Self. New York: Cambridge University Press, 1981.

FRASCARA, J. Diseño Gráfico para la Gente. Buenos Aires: Ediciones Infinito, 2000.

Guia Global: Cidade Amiga do Idosos/ Organização Mundial da Saúde. Brasília:

Organização Pan-Americana da Saúde, 2007.

GOLDENBERG, M. A Bela Velhice. 1. Ed - Rio de Janeiro: Record, 2013.

Cuidar da casa pode ser seu melhor objetivo. Casa e Jardim: Ed.

Globo, 2015. Disponível em: <http://revistacasaejardim.globo.com/Casa-eJardim/noticia/2014/11/casa-de-outra-perspectiva.html> Acesso em: 15 jul. 2015. (a)

Disponível em: <http://revistacasaejardim.globo.com/Casa-eJardim/Colunistas/Terapia-da-casa/noticia/2015/07/novas-casas-para-novosidosos.html> Acesso em: 21 jul. 2015. (b)

HALBWACHS, M. A Memória Coletiva. São Paulo: Vértice, 1990.

HELPAGE INTERNATIONAL. Global AgeWatch Index 2015. Disponível em: $<$ http://www.helpage.org/global-agewatch/population-ageing-data/global-rankingstable/> Acesso em: 9 set. 2015.

IBGE, Censos demográficos e PNADs, diversos anos.

INTERNATIONAL LONGEVITY CENTER BRAZIL. Active Ageing: A Policy Framework in Response to the Longevity Revolution. Disponível em: <http://www.ilcbrazil.org/wpcontent/uploads/2015/07/ActiveAgeingPolicyFramework_2015_v1.1.pdf>, acesso em 20 jul. 2015.

MCKRACKEN, G. Cultura e Consumo. Rio de Janeiro: Mauad, 2003.

MOTLIS, J. El Dado de la Vejez y su Seis Caras: Como Interpretar el acontecer del Envejecimento. Madrid: Altalena, 1985.

MORAES, E. N., CINTRA, M. T. G. Avaliação Geriátrica. In: MALLOY-DINIZ, L. F. I.; FUENTES, D.; COSENZA, R. M. (Org.) Neuropsicologia do Envelhecimento: Uma Abordagem Multidimensional. Porto Alegre: Artmed, 2013.

NERI, A. L. Paradigmas Contemporâneos sobre o Desenvolvimento Humano em Psicologia e em Sociologia. In: NERI, A. L (ORG.) Desenvolvimento e Envelhecimento: Perspectivas Biológicas, Psicológicas e Sociológicas. 3ed. Campinas, SP: Papirus, 2007. 
NUNES, V. Larrousse da Terceira Idade. São Paulo: Larrousse do Brasil, 2003.

RIBEIRO, A. M.; COSENZA, R. M. Envelhecimento Normal do Sistema Nervoso. In MALLOY-DINIZ, L. F. I.; FUENTES, D.; COSENZA, R. M. Neuropsicologia do Envelhecimento: Uma Abordagem Multidimensional. Porto Alegre: Artmed, 2013.

WORLD HEALTH ORGANIZATION (WHO). World Report on Ageing and Health. Geneva. 2015 